\title{
Improved Prophet Inequalities for Combinatorial Welfare Maximization with (Approximately) Subadditive Agents
}

\author{
Hanrui Zhang \\ Duke University, Durham, NC, USA \\ hrzhang@cs.duke.edu
}

\begin{abstract}
We give a framework for designing prophet inequalities for combinatorial welfare maximization. Instantiated with different parameters, our framework implies (1) an $O(\log m / \log \log m)$-competitive prophet inequality for subadditive agents, improving over the $O(\log m)$ upper bound via item pricing, (2) an $O(D \log m / \log \log m)$-competitive prophet inequality for $D$-approximately subadditive agents, where $D \in\{1, \ldots, m-1\}$ measures the maximum number of items that complement each other, and (3) as a byproduct, an $O(1)$-competitive prophet inequality for submodular or fractionally subadditive (a.k.a. XOS) agents, matching the optimal ratio asymptotically. Our framework is computationally efficient given sample access to the prior and demand queries.
\end{abstract}

2012 ACM Subject Classification Theory of computation $\rightarrow$ Stochastic approximation; Theory of computation $\rightarrow$ Algorithmic game theory and mechanism design

Keywords and phrases Prophet Inequalities, Combinatorial Welfare Maximization, (Approximate) Subadditivity

Digital Object Identifier 10.4230/LIPIcs.ESA.2020.82

Funding Hanrui Zhang: Supported by NSF award IIS-1814056.

Acknowledgements The author thanks Yuan Deng, Kamesh Munagala, and anonymous reviewers for helpful feedback.

\section{Introduction}

Prophet inequalities are a classical topic in stopping theory. The problem is neat and natural: an agent plays a game, where there are $n$ boxes, each containing a reward (e.g., some amount of cash). The agent cannot see through the boxes to know precisely the amounts of cash inside each box. However, she has the prior knowledge that the amounts are drawn independently for each box, and fortunately, knows the distributions according to which the amounts are drawn. Now nature opens the boxes one by one. Upon seeing the inside of each box, the agent gets to make a choice: she can either (1) take the cash in the box and leave, or (2) let the current box expire (which means she gains nothing from the current box and it disappears), in which case the game proceeds with the remaining boxes. What is the maximum expected amount of cash the agent can get, and how to achieve that?

Quite surprisingly, the agent can guarantee half the amount of reward that a prophet is able to get, who sees through the boxes and therefore always picks the box with the largest reward [21, 22]. Moreover, the agent can achieve this by executing a simple threshold-based protocol: accept the first box containing a reward exceeding a pre-calculated amount. The existence of such 2-approximate protocols lead to the name "prophet inequalities."

Prophet inequalities were recently rediscovered in computer science and economics. Since then, they have been drawing increasing interest in both fields. Hajiaghayi et al. [18] observe the connection between prophet inequalities and a pricing problem in auctions. They formulate the problem in the following equivalent way: a seller has an indivisible item to 
sell. $n$ buyers arrive one by one, each of which has a value for the item, drawn independently from a distribution known to the seller. When a buyer arrives, the seller learns the value of the buyer (or otherwise negotiates with the buyer), and decides whether to sell the item to the buyer. The buyer then leaves forever (with the item if sold to the buyer). The goal of the seller is to maximize the utility of the buyer who receives the item, or just the total utility (i.e., the welfare) of all buyers, since all other buyers have utility 0 . Here, each buyer corresponds to a box in the classical formulation, and the value of the buyer represents the reward in the box. A threshold-based protocol can then be translated directly into a take-it-or-leave-it offer - a buyer receives the item (i.e., she buys) iff her value exceeds the pre-calculated price, and so buying is preferred to not buying.

Given this connection, various forms of auctions have been considered in the prophet inequality context (see, e.g., the recent survey by Lucier [23]). Examples include (1) the case where the seller has $k$ identical items to sell and each buyer wants only one of them (or equivalently, up to $k$ boxes can be accepted) [18], (2) the setting with a knapsack style constraint, requiring that the total "weight" of sellers who get an item cannot exceed 1 [17], and (3) the setting where $m$ possibly distinct items are available for sale, and each agent has a combinatorial (as opposed to additive) valuation function assigning every subset of items a value [16]. The third setting, known as combinatorial welfare maximization, appears particularly interesting and general, as it nicely captures the potentially complex interaction between items. For instance, a Coke and a Pepsi substitute each other, in the sense that having a Coke or a Pepsi probably gives one roughly the same utility (say 1), whereas having both likely gives strictly less utility than the sum, 2 , of the former values, since one can only drink so much at a time.

In this paper, we consider combinatorial welfare maximization in the prophet inequality context. We begin our investigation with subadditive (also known as complement-free) agents, who regard items only as substitutes but not complements to each other. From there, we generalize our results to accommodate valuations that are approximately subadditive, which have remained largely unexplored even in offline environments.

\subsection{Our Contributions}

\subsubsection{Current Landscape of the Problem}

Feldman et al. [16] were the first to explicitly study combinatorial welfare maximization with rich valuations in the prophet inequality context. They give an existential 2-approximate protocol and a computationally efficient $(2 e) /(e-1)$-approximate protocol when agents are submodular or fractionally subadditive (which are strict subclasses of subadditive valuations). Dütting et al. [10] propose a powerful framework, unifying a number of prophet inequalities, and yielding a computationally efficient 2-approximate protocol for the same class of valuations, which is optimal given a lower bound inherited from the single-item setting. These bounds, through a standard approximation result, extend directly to subadditive agents with a loss of factor $O(\log m)$, where $m$ is the number of items. The best known lower bound for subadditive agents, however, is again 2, leaving a huge gap in between. This gap, as acknowledged by Feldman et al. [16] and Dütting et al. [10], raises a curious question: 


\subsubsection{The Logarithmic Barrier for Subadditive Agents}

The above question did not have an immediate answer. For subadditive agents, all existing results essentially build on the same argument: one first computes prices that $O(\log m)$ approximately "support" the optimal allocation. Such prices have the property, that if one posts the prices on the items, and let agents, one by one in some arbitrary order, purchase their utility-maximizing bundles of items, then the resulting allocation $O(\log m)$ approximately maximizes the expected welfare. With these prices, one can implement an $O(\log m)$-approximate threshold-based protocol, by running a posted-price auction with the supporting prices for each arriving agent, and allocating the set of items purchased to the agent. The bottleneck of this approach is that the $O(\log m)$ factor of approximate supporting prices is tight: there are subadditive valuations for which no $o(\log m)$-approximate supporting prices exist [2]. Therefore, any protocol relying on supporting prices (including all currently existing results) cannot possibly give better ratios than $O(\log m)$. In fact, given the tightness of this $O(\log m)$ factor, one may even suspect that the right ratio for subadditive agents is precisely $\Theta(\log m)$. We show that this is not the case.

\subsubsection{A Sublogarithmic Prophet Inequality for Subadditive Agents}

We give a framework for designing prophet inequalities for combinatorial welfare maximization, which implies an $O(\log m / \log \log m)$-approximate prophet inequality for subadditive agents, breaking the foregoing logarithmic barrier. Our framework is computationally efficient given: (1) sample access to the prior distributions, and (2) demand queries to the sample valuations. Unlike previous results, our protocol is not based on pricing items via approximately supporting prices and running sequential auctions - which enables the protocol to bypass the obstacle discussed above, at the cost of losing incentive compatibility. ${ }^{1}$ As a byproduct, we show that our framework, instantiated with different parameters, also gives an $O(1)$-approximately optimal prophet inequality for submodular or fractionally subadditive agents. Our approach provides an alternative view of combinatorial welfare maximization in the prophet inequality context, which may be of independent interest.

Very recently, in independent work, Dütting et al. [11] give an $O(\log \log m)$-approximate prophet inequality for combinatorial welfare maximization with subadditive agents using radically different techniques from ours. Their result is a major breakthrough in the research of prophet inequalities for combinatorial welfare maximization. In particular, the work of Dütting et al. significantly improves over our main result in that (1) they "truely" improve the approximation ratio from $O(\log m)$ all the way to $O(\log \log m)$, and (2) their approach is based on pricing individual items, which shows that it is possible to beat $O(\log m)$ using item-pricing schemes, which are incentive-compatible.

\subsubsection{Generalizing to Approximate Subadditivity}

Utilizing the same framework, we give a family of parameters, which imply an $O(D \log m / \log \log m)$-approximate prophet inequality when agents have valuations with superadditive width at most $D>0$ (see Definition 1). As a corollary, we obtain an $O(D \log m / \log \log m)$-approximation algorithm for the offline combinatorial welfare maximization problem with the same class of valuations. Here, $D$ roughly measures the maximum

\footnotetext{
${ }^{1}$ In some scenarios, incentive incompatibility is not an issue - one such example is a government agency allocating resources among projects arriving online.
} 
number of items that may complement each other, and generalizes the notion of subadditivity. In particular, valuations with superadditive width 0 are precisely valuations that are subadditive. The implication of the above bound is twofold: concretely, to our knowledge, this is the first nontrivial prophet inequality for valuations that are approximately subadditive; conceptually, the existence of parameters leading to this bound demonstrates the capacity of the parametrized framework we propose. We remark that while Feldman et al. [16] and Dütting et al. [10] present results of a similar flavor, their bounds are based on the Maximum-over-Positive-Hypergraph (MPH) hierarchy [15], which does not directly model approximate subadditivity, and thus is incompatible with the goal of this paper.

\subsection{Technical Overview}

We present a parametrized protocol, which works by rounding online a standard LP relaxation of the welfare maximization problem. The protocol is inspired by the two-stage offline rounding procedure by Dobzinski et al. [9], which works roughly as follows:

1. Compute a distribution over sets of items for each agent. These distributions together satisfy: (1) each item in expectation goes to at most 1 agent, and (2) the total expected value enjoyed by all agents is maximized.

2. For each agent $i$, draw $i$ 's tentative set of items according to $i$ 's distribution. Note that after this step, an item can appear in multiple agents' tentative sets.

3. Break ties for items, by independently allocating each item $j$ to one of the agents whose tentative sets contain $j$, uniformly at random.

Dobzinski et al. [9] show that the above procedure outputs an $O(\log m)$-approximation for the combinatorial welfare maximization problem when agents are subadditive, and Feige [14] further proves a tighter bound of $O(\log m / \log \log m)$.

Intuitively, we wish to carry out Dobzinski's rounding procedure online. One main difficulty, however, is to deal with incomplete information, since when handling one agent, the protocol does not know the actual valuation of any agent yet to arrive. As a result, it is impossible to round online the solution to the LP with respect to all agents' actual valuations. To this end, we create a partially fictitious LP for each agent, by chaining this agent's actual valuation (which becomes available to the protocol upon the agent's arrival) with independently drawn dummy valuations for all other agents. By doing this, each agent is intuitively playing against the average case configuration of all other agents. Moreover, in each agent's fictitious LP, her share of the fractional allocation gives her precisely the expected value she would get in the actual optimal fractional allocation. So if we can round the fictitious LP solutions with mild loss, the resulting welfare will in fact be approximately optimal.

To achieve this, we need to deal with another difficulty, i.e., instead of breaking ties for each item simultaneously with all the tentative sets available, we now have to irrevocably decide which items are being allocated to each agent immediately upon his arrival, before seeing the valuations of all agents yet to arrive. And still, we need to make sure no item is allocated twice. This rules out the possibility of the independent uniform tie-breaking performed in Step 3 of Dobzinski et al.'s rounding procedure, which is crucial in the proofs for the approximation ratios in both papers. ${ }^{2}$ To overcome this issue, we break ties in a correlated way, which pairs gracefully with (approximate) subadditivity. Roughly speaking,

2 With some additional tricks one can simulate online the uniformity and independence conditions required in those papers, but even then the online version of Feige's argument works only for exactly subadditive valuations, and fails for approximately subadditive ones. 
we first draw a (not necessarily uniformly) random integer $r$ from $\{1, \ldots, n\}$, where $n$ is the number of agents. We then partition each agent's tentative set into $n$ subsets according to the number of times each item has appeared in some agent's tentative set so far, and give the agent all items that have appeared exactly $r$ times. The intuition is that, if the valuations are subadditive, we then know that the sum of the values of these subsets is at least the value of the tentative set itself, which gives us a way to relate the expected value of the allocated subset to the value of the tentative set. Also, no item is possibly allocated twice, since each item can appear the $r$-th time only once throughout the procedure. Given the above, by choosing the distribution of $r$ and other parameters in different ways, the parametrized protocol yields the desired bounds for subadditive, approximately subadditive, and fractionally subadditive valuations respectively.

\subsection{Additional Related Work}

Kleinberg and Weinberg [20] study the setting where possible combinations of boxes that can be accepted satisfy a matroid constraint. Dütting and Kleinberg [12] consider polymatroids, generalizing the above setting. Rubinstein and Singla [25] further consider subadditive reward functions. Their setting, while also being combinatorial, is different from combinatorial welfare maximization considered in this paper.

Another line of research consider revenue maximization in the prophet inequality context. We list a few results here. Blumrosen and Holenstein [3] give a constant factor protocol in the single-item setting. When agents are unit-demand - that is, they only want a single item - Chawla et al. [5] give constant factor posted-price policies. Cai and Zhao [4] study truthful policies for combinatorial auctions with subadditive agents. Their goal, however, is to maximize the revenue of the protocol, instead of the welfare as we consider.

Prior to Feldman et al., Chawla et al. [6] and Alaei [1] consider welfare maximization with unit-demand agents. Cohen-Addad et al. [8] further show that dynamic pricing achieves optimal welfare for unit-demand agents. Ehsani et al. [13] show that the ratio improves to $e /(e-1)$ for combinatorial welfare maximization with submodular or fractionally subadditive agents, if agents arrive in a random order.

\section{Preliminaries}

Throughout the paper, we use $n$ to denote the number of agents, and $m$ the number of items. In general, we use $i$ as the index of an agent, and $j$ as the index of an item.

\subsection{Combinatorial Valuations}

A combinatorial valuation function $f: 2^{[m]} \rightarrow \mathbb{R}^{+}$maps any subset $S$ of the ground set $[m]=\{1,2, \ldots m\}$ to a nonnegative real number $f(S)$. In this paper, we consider valuation functions that are monotone: $f$ is monotone iff for any $S \subseteq T \subseteq[m], f(S) \leq f(T)$. The following subclasses of valuation functions are considered or helpful for our purposes:

- subadditive: $f$ is subadditive iff for any $S, T \subseteq[m], f(S)+f(T) \geq f(S \cup T)$.

- additive valuations: $f$ is additive iff for any disjoint $S, T \subseteq[m], f(S)+f(T)=f(S \cup T)$.

- submodular valuations: $f$ is submodular iff for any $S, T \subseteq[m], f(S)+f(T) \geq f(S \cup T)+$ $f(S \cap T)$.

- fractionally subadditive (or XOS) valuations: $f$ is fractionally subadditive iff there exist additive valuations $c^{1}, \ldots, c^{\ell}$, such that for any $S \subseteq[m], f(S)=\max _{k \in[\ell]} c^{k}(S)$. Each such additive valuation $c^{k}$ is called a clause. 
It is known that every additive valuation is submodular, every submodular valuation is fractionally subadditive, and every fractionally subadditive function is subadditive. Beyond subadditive valuations, we also consider valuations of limited superadditivity, parametrized by the superadditive width, defined below.

- Definition 1 (Superadditive Width [7]). Fix a ground set $M=[m]$. A set $T \subseteq M$ is superadditive w.r.t. a valuation function $f$, if there exists $S \subseteq M$, such that

$$
f(S \mid T)>\max _{T^{\prime} \subsetneq T} f\left(S \mid T^{\prime}\right),
$$

where $f(A \mid B):=f(A \cup B)-f(B)$ is the marginal value of $A$ given $B$ for any two sets $A, B \subseteq M$. The superadditive width of $f$ is defined to be the size of the largest superadditive set, i.e.,

$$
\operatorname{SAW}(f):=\max \{|T| \mid T \text { is superadditive w.r.t. } f\} .
$$

In words, the definition says that the superadditive width of a valuation is the size of the largest set, which provides strictly more marginal value for another set, than any of its strict subsets. Intuitively, the smaller this quantity is, the closer a valuation is to being subadditive. In particular, any subadditive valuation has superadditive width 0 .

\subsection{Problem Formulation}

We formulate the problem in the following way: There are $n$ agents and $m$ items, and a prior $\mathcal{F}=\mathcal{F}_{1} \times \cdots \times \mathcal{F}_{n}$ for the valuations of the agents over the items. All agents are monotone and subadditive (resp., fractionally subadditive), i.e., for any agent $i$, any valuation function $f_{i}$ in the support of $\mathcal{F}_{i}$ is monotone and subadditive (resp., fractionally subadditive). Agents arrive one by one in an adversarial order. When agent $i$ arrives, we see the realization $f_{i} \sim \mathcal{F}_{i}$ of her valuation (through query oracles discussed below), and must allocate irrevocably some items to the agent. The agent then takes the items and departs, and all items allocated to the agent become unavailable to subsequently arriving agents.

The goal is to maximize the expected (over the realization of the valuations and the randomness of the protocol) welfare of all agents. In particular, we wish to compete against the offline optimal allocation (i.e., the prophet), which has unlimited computational power, and knows beforehand the realization of all agents' valuations $\left\{f_{i}\right\}_{i}$. The competitive ratio is defined to be the ratio between the expected welfare (denoted OPT) of the offline optimal allocation $^{3}$ and the expected welfare produced by the online protocol.

\subsection{Oracle Access to Valuation Functions}

The representation of a combinatorial valuation function may be exponentially large in the number of items $m$. Given this complexity, it is standard to assume that the protocol may access valuation functions only through query oracles. In particular, the following two types of queries are commonly allowed (see, e.g., $[14,16,10])$ :

- value queries: given a valuation function $f$ and a set $S$, return the value of $S, f(S)$.

- demand queries: given a valuation function $f$ and prices $\left\{p_{j}\right\}_{j \in[m]}$, return a utilitymaximizing set (i.e., a demand set) with respect to $f$ under the given prices. That is, the query returns a set $S$ that maximizes $f(S)-\sum_{j \in S} p_{j}$.

In this paper, we assume the protocol has access to both kinds of queries.

3 Note that the offline optimal allocation may be hard to find, computationally and / or information theoretically. 


\subsection{The Welfare Maximizing LP}

The following LP relaxation of the welfare maximization problem has been considered in $[9,24,14]$ :

$$
\begin{array}{rll}
\operatorname{maximize} & \sum_{i \in[n]} \sum_{S \subseteq[m]} x_{i, S} f_{i}(S) & \\
\text { s.t. } & \sum_{S \subseteq[m]} x_{i, S} \leq 1 & \forall i \in[n] \\
& \sum_{i \in[n], S \subseteq[m]: j \in S} x_{i, S} \leq 1 & \forall j \in[m] \\
& x_{i, S} \geq 0 & \forall i \in[n], S \subseteq[m] .
\end{array}
$$

One may interpret the LP in the following way: $x_{i, S}$ stands for the probability that agent $i$ receives bundle $S$. The objective is therefore the total expected value of all agents, which is the expected welfare. The first constraint requires that each agent $i$ receives at most 1 bundle, and the second requires that each item goes to at most 1 agent, in expectation.

It is known (see, e.g., $[9,24]$ ) that the above LP can be solved with polynomially many value and demand queries to $f_{1}, \ldots, f_{n}$. Let $x_{i, S}\left(\left\{f_{i^{\prime}}\right\}_{i^{\prime}}\right)$ (parameters omitted when clear from the context) denote the value of variable $x_{i, S}$ in the optimal solution to the LP with respect to valuation functions $\left\{f_{i^{\prime}}\right\}_{i^{\prime}} \cdot{ }^{4}$ Given $\mathcal{F}$, denote the expected value enjoyed by agent $i$ in this optimal solution by

$$
\mathrm{LP}_{i}:=\mathbb{E}_{\left\{f_{i^{\prime}}\right\}_{i^{\prime}} \sim \mathcal{F}}\left[\sum_{S \subseteq[m]} x_{i, S}\left(\left\{f_{i^{\prime}}\right\}_{i^{\prime}}\right) \cdot f_{i}(S)\right]
$$

and the optimal objective value by

$$
\mathrm{LP}:=\sum_{i \in[n]} \mathrm{LP}_{i}=\mathbb{E}_{\left\{f_{i^{\prime}}\right\}_{i^{\prime}} \sim \mathcal{F}}\left[\sum_{i \in[n], S \subseteq[m]} x_{i, S}\left(\left\{f_{i^{\prime}}\right\}_{i^{\prime}}\right) \cdot f_{i}(S)\right] .
$$

Note that for any prior $\mathcal{F}$, we always have LP $\geq$ OPT. We will use the welfare maximizing LP and its solution as a building block of our framework in a blackbox manner.

\section{The Framework}

In this section, we present our general framework, in the form of a parametrized protocol, for designing prophet inequalities for combinatorial welfare maximization. The framework works for any arrival order of agents, but for ease of presentation, we assume agents arrive according to their indices. That is, agent 1 arrives first, followed by agent 2 , etc. We also let $f_{-i}:=\left(f_{1}, \ldots, f_{i-1}, f_{i+1}, \ldots, f_{n}\right)$ (i.e., $f_{-i}$ denotes the valuations of all agents but $i$ ) and use $g_{-i}$ and $\mathcal{F}_{-i}$ similarly. Below is our parametrized protocol:

1. For each item $j$, initialize counter $c_{j} \leftarrow 0$.

2. Draw positive integer $r \in[n]$, where $\operatorname{Pr}[r=k]=p_{k}$ for any $k \in[n]$, and $\left\{p_{k}\right\}_{k \in[n]}$ are parameters of the protocol.

3. Upon agent $i$ 's arrival:

a. Let $f_{i}$ be agent $i$ 's realized valuation; draw dummy valuations $g_{-i} \sim \mathcal{F}_{-i}$ for all other agents. $^{5}$

\footnotetext{
4 If there are multiple such solutions, let $\left\{x_{i, S}\left(\left\{f_{i^{\prime}}\right\}_{i^{\prime}}\right)\right\}_{i, S}$ be the one produced by the efficient LP solving algorithm which we use as a subroutine of the protocol.

5 Note that we abuse notation here, so for any $i_{1} \neq i_{2}, g_{-i_{1}}$ and $g_{-i_{2}}$ are independent - they are not different parts of a same group of valuations.
} 
b. Solve the welfare maximizing LP with valuations $\left(f_{i}, g_{-i}\right)$, and let $\left\{x_{i^{\prime}, S}\left(f_{i}, g_{-i}\right)\right\}_{i^{\prime}, S}$ be the solution.

c. Now view $\left\{x_{i, S}\right\}_{S}$ as a distribution over sets of items. ${ }^{6}$ Draw set $S_{i} \sim\left\{x_{i, S}\right\}_{S}$ from this distribution, where for any $S, \operatorname{Pr}\left[S_{i}=S\right]=x_{i, S}$. We say agent $i$ demands set $S_{i}$.

d. For each item $j \in S_{i}$, let $c_{j} \leftarrow c_{j}+1$ (i.e., increase the counter for item $j$ ). For any $k \in[n]$, let $S_{i}^{k}$ be the set of items in $S_{i}$ whose counters are exactly $k$ right after demanded by agent $i$.

e. With probability $q$, where $q$ is a parameter of the protocol, serve agent $i$ by giving $i$ all items in $S_{i}^{r}$ (i.e., item $j$ goes to agent $i$ iff agent $i$ is chronologically the $r$-th agent demanding item $j$ and being served); otherwise, for each item $j \in S_{i}$, let $c_{j} \leftarrow c_{j}-1$ (i.e., undo the increase for all items demanded by $i$ ).

For any agent $i$, let $\operatorname{ser}_{i}=\mathbb{I}[i$ is served $]$ be the indicator variable that $i$ is served in Step 3(e). Note that the above protocol never allocates an item to more than one agent, since once $r$ is fixed, at most one agent can be the $r$-th demanding an item. As a result, the protocol always produces a valid allocation.

We now present a meta-analysis for the above protocol, which yields a parameterdependent welfare guarantee. In later sections, we will show how one can instantiate the protocol by setting different parameters, so that the general guarantee realizes into specific bounds for the respective classes of valuations of interest.

- Theorem 2. Let $u_{i}$ be the value enjoyed by agent $i$. For each agent $i$, fixing $f_{i}$ and $S_{i}$, the above protocol guarantees agent $i$ expected value

$$
\mathbb{E}_{R_{i}}\left[u_{i} \mid f_{i}, S_{i}\right]=q \cdot \sum_{k \in[n]} p_{k} \cdot \mathbb{E}_{R_{i}}\left[f_{i}\left(S_{i}^{k}\right)\right]
$$

where $R_{i}$ summarizes all the randomness other than $f_{i}, g_{-i}$ and $S_{i}$, both from the protocol and from the realization of the valuations.

Proof. The theorem is essentially a claim regarding independence of random variables. Recall that for any agent $i^{\prime}, \operatorname{ser}_{i^{\prime}}=\mathbb{I}\left[i^{\prime}\right.$ is served $]$. Fixing $f_{i}$ and $S_{i}$, $i^{\prime}$ s expected value can be written as

$$
\mathbb{E}_{R_{i}}\left[u_{i} \mid f_{i}, S_{i}\right]=\mathbb{E}_{R_{i}}\left[\operatorname{ser}_{i} \cdot \sum_{k \in[n]} \mathbb{I}[r=k] \cdot f_{i}\left(S_{i}^{k}\right) \mid f_{i}, S_{i}\right] .
$$

$R_{i}$ here summarizes $\left\{\left(f_{i^{\prime}}, g_{-i^{\prime}}, S_{i^{\prime}}\right)\right\}_{i^{\prime} \neq i}$, the choice of $r$, and whether $i^{\prime}$ is served for all $i^{\prime}$ (including $i^{\prime}=i$ ). To simplify this, first observe that the conditioning can be removed, because the randomness involved in the expectation, $R_{i}$, is independent of $f_{i}$ and $S_{i}$. In particular, $\left\{f_{i^{\prime}}\right\}_{i^{\prime} \neq i}$ are independent of $f_{i}$ because $\mathcal{F}$ is a product distribution. Moreover, the three factors within the expectation are independent, because whether $i$ is served depends only on the coin flipping in Step 3(e) when agent $i$ arrives, the choice of $r$ depends only on the random draw in Step 2, and fixing $S_{i}, f_{i}\left(S_{i}^{k}\right)$ depends only on the realization of $\left\{f_{i^{\prime}}\right\}_{i^{\prime}<i}$ and the random bits of the protocol dealing with the agents arriving before $i$. Given the above, we have

${ }^{6}$ It is possible that $\sum_{S} x_{i, S}<1$, in which case with probability $1-\sum_{S} x_{i, S}, S_{i}=\emptyset$. 


$$
\begin{aligned}
\mathbb{E}_{R_{i}}\left[u_{i} \mid f_{i}, S_{i}\right] & =\mathbb{E}_{R_{i}}\left[\operatorname{ser}_{i} \cdot \sum_{k \in[n]} \mathbb{I}[r=k] \cdot f_{i}\left(S_{i}^{k}\right)\right] \\
& =\operatorname{Pr}\left[\operatorname{ser}_{i}=1\right] \cdot \sum_{k \in[n]} \operatorname{Pr}[r=k] \cdot \mathbb{E}_{\left\{\left(f_{i^{\prime}}, g_{-i^{\prime}}, S_{i^{\prime}}, \operatorname{ser}_{i^{\prime}}\right)\right\}_{i^{\prime}<i}}\left[f_{i}\left(S_{i}^{k}\right)\right] \\
& =q \cdot \sum_{k \in[n]} p_{k} \cdot \mathbb{E}_{R_{i}}\left[f_{i}\left(S_{i}^{k}\right)\right] .
\end{aligned}
$$

Before proceeding to the specific instantiations of the protocol, we note the following high-level interpretation of the above parameter-dependent bound, which provides important intuition and leads to our choices of parameters for different classes of valuations. For concreteness, suppose agents are subadditive. First observe that the share of agent $i$ in the optimal solution of the welfare maximizing LP is

$$
\begin{aligned}
\operatorname{LP}_{i} & =\mathbb{E}_{\left\{f_{i^{\prime}}\right\}_{i^{\prime}} \sim \mathcal{F}}\left[\sum_{S} x_{i, S}\left(\left\{f_{i^{\prime}}\right\}_{i^{\prime}}\right) \cdot f_{i}(S)\right] \\
& =\mathbb{E}_{\left(f_{i}, g_{-i}\right) \sim \mathcal{F}, S_{i} \sim\left\{x_{i, S}\left(f_{i}, g_{-i}\right)\right\}_{S}}\left[f_{i}\left(S_{i}\right)\right]=\mathbb{E}_{f_{i}, S_{i}}\left[f_{i}\left(S_{i}\right)\right] .
\end{aligned}
$$

On the other hand, since $\left\{S_{i}^{k}\right\}_{k \in[n]}$ is a partition of $S_{i}$, by the subadditivity of $f_{i}$, we always have

$$
\sum_{k \in[n]} \mathbb{E}_{R_{i}}\left[f_{i}\left(S_{i}^{k}\right)\right] \geq \mathbb{E}_{R_{i}}\left[f_{i}\left(S_{i}\right)\right]=f_{i}\left(S_{i}\right) .
$$

So hypothetically, if somehow we were able to set $q=1$ and $p_{k}=1$ for every $k \in[n]$ simultaneously (which is of course impossible), then fixing any choice of $f_{i}$ and $S_{i}$, the above protocol would yield at least $f_{i}\left(S_{i}\right)$ as the expected value for agent $i$. Further taking expectation over $f_{i}$ and $S_{i}$, this would imply a 1 -approximate prophet inequality against the welfare maximizing LP.

In reality, however, one cannot set $p_{k}$ to be large for all $k$ simultaneously. In fact, these probabilities must sum to 1 . Still, our goal is to guarantee a decent fraction of $f_{i}\left(S_{i}\right)$ for any choice of $f_{i}$ and $S_{i}$. This is possible when, for example, the total value of $S_{i}, f_{i}\left(S_{i}\right)$, concentrates in relatively few entries among its $n$ parts, $\left\{\mathbb{E}_{R_{i}}\left[f_{i}\left(S_{i}^{k}\right)\right]\right\}_{k \in[n]}$. In such cases, we can let $r$ be uniformly distributed over the indices of these entries, leading to a competitive ratio proportional to the number of such heavy entries. The key step here is analyzing the distribution of $f_{i}\left(S_{i}\right)$ into $\left\{f_{i}\left(S_{i}^{k}\right)\right\}_{k \in[n]}$, over the randomness in $R_{i}$. We will further develop the above intuition in later sections.

\section{Warmup: the Case of Fractionally Subadditive Agents}

We start with the relatively simple case of fractionally subadditive agents, the analysis for which provides tools for the more involved cases to be handled in later sections. Throughout this section, we consider the following choice of parameters: $p_{1}=1, p_{k}=0$ for any $1<k \leq n$, and $q=1 / 2$. That is, each agent is served with probability $1 / 2$, and whenever an agent is served, he receives all the items demanded which are not yet taken. We now analyze our protocol with the above parameters for fractionally subadditive agents.

- Theorem 3. The protocol in Section 3 with $p_{1}=1, p_{k}=0$ for any $1<k \leq n$, and $q=1 / 2$ is 4-competitive when agents are fractionally subadditive. 
Proof. In light of Theorem 2, we only need to show that for any agent $i$, fixing $f_{i}$ and $S_{i}$,

$$
\mathbb{E}_{R_{i}}\left[f_{i}\left(S_{i}^{1}\right)\right] \geq \frac{1}{2} f_{i}\left(S_{i}\right) .
$$

The theorem then follows by plugging the above into Theorem 2 and taking expectation over $f_{i}$ and $S_{i}$, which yields

$$
\mathbb{E}_{f_{i}, S_{i}}\left[\mathbb{E}_{R_{i}}\left[u_{i} \mid f_{i}, S_{i}\right]\right]=\mathbb{E}_{f_{i}, S_{i}}\left[\frac{1}{2} \mathbb{E}_{R_{i}}\left[f_{i}\left(S_{i}^{1}\right)\right]\right] \geq \frac{1}{4} \mathbb{E}_{f_{i}, S_{i}}\left[f_{i}\left(S_{i}\right)\right]=\frac{1}{4} \operatorname{LP}_{i} .
$$

Summing over the agents, we get the following lower bound on the expected welfare:

$$
\mathbb{E}\left[\sum_{i \in[n]} u_{i}\right] \geq \frac{1}{4} \sum_{i \in[n]} \mathrm{LP}_{i}=\frac{1}{4} \mathrm{LP} \geq \frac{1}{4} \mathrm{OPT},
$$

where the expectation is over all the randomness, both from the protocol and from the realization of agents' valuations. This gives precisely the desired competitive ratio of 4 . The rest of the proof is dedicated to establishing (1) when $i, f_{i}$ and $S_{i}$ are fixed.

Recall the following property of fractionally subadditive functions.

- Lemma 4. For fractionally subadditive $f$ and any set of items $S$, let $T$ be such that for any $j \in S, j \in T$ with probability at least $p$. Then

$$
\mathbb{E}[f(T)] \geq p \cdot f(S) .
$$

While this is sometimes considered standard, we give below a quick proof for completeness.

Proof. Let $c^{k}$ be the clause of $f$ such that

$$
f(S)=c^{k}(S)=\sum_{j \in S} c^{k}(\{j\}) .
$$

By the fractional subadditivity of $f$, for any $S^{\prime} \subseteq S$,

$$
f\left(S^{\prime}\right) \geq c^{k}\left(S^{\prime}\right) .
$$

This is in particular true for $T$, which implies

$$
\begin{aligned}
\mathbb{E}[f(T)] & \geq \mathbb{E}\left[c^{k}(T)\right] \\
& =\sum_{j \in S} \mathbb{E}\left[\mathbb{I}[j \in T] \cdot c^{k}(\{j\})\right] \\
& =\sum_{j \in S} \operatorname{Pr}[j \in T] \cdot c^{k}(\{j\}) \\
& \geq p \cdot \sum_{j \in S} c^{k}(\{j\}) \\
& =p \cdot f(S) .
\end{aligned}
$$

Given Lemma 4, the plan is to show that each item $j$ in $S_{i}$ appears in $S_{i}^{1}$ with probability at least $1 / 2$. That is, with probability at least $1 / 2$, no agent $i^{\prime}<i$ demands item $j$ and gets served simultaneously. To see why this is true, consider the unconditional distribution of the set $S_{i^{\prime}}$ demanded by any agent $i^{\prime}$. Let $y_{i^{\prime}, S}=\operatorname{Pr}\left[S_{i^{\prime}}=S\right]$ be the probability that agent $i^{\prime}$ demands set $S$, over $f_{i^{\prime}}, g_{-i^{\prime}}$, and the random bits of the protocol. Note that $y_{i^{\prime}, S}$ is not random, and depends only on the prior $\mathcal{F}$. We first show that $\left\{y_{i^{\prime}, S}\right\}_{i^{\prime}, S}$ form a feasible solution to the welfare maximizing LP, regardless of the actual valuations of the agents. This is well-defined, since fixing $n$ and $m$, the precise values of sets do not appear in any constraint of the welfare maximizing LP. 
- Lemma 5. $\left\{y_{i^{\prime}, S}\right\}_{i^{\prime}, S}$ satisfy:

- for any agent $i^{\prime} \in[n], \sum_{S \subseteq[m]} y_{i^{\prime}, S} \leq 1$,

- for any item $j \in[m], \sum_{i^{\prime} \in[n], S \subseteq[m]: j \in S} y_{i^{\prime}, S} \leq 1$, and

- for any $i^{\prime} \in[n], S \subseteq[m], y_{i^{\prime}, S} \geq 0$.

Proof. Observe that according to the protocol, for any $i^{\prime}, S$,

$$
y_{i^{\prime}, S}=\mathbb{E}_{f_{i^{\prime}} \sim \mathcal{F}_{i^{\prime}}, g_{-i^{\prime}} \sim \mathcal{F}_{-i^{\prime}}}\left[x_{i^{\prime}, S}\left(f_{i^{\prime}}, g_{-i^{\prime}}\right)\right]=\mathbb{E}_{\left\{f_{i^{\prime \prime}}\right\}_{i^{\prime \prime}} \sim \mathcal{F}}\left[x_{i, S}\left(\left\{f_{i^{\prime \prime}}\right\}_{i^{\prime \prime}}\right)\right] .
$$

In other words, $y_{i^{\prime}, S}$ is the expected value of the variable $x_{i^{\prime}, S}$ in the optimal solution to the welfare maximizing LP, when valuations are distributed according to prior $\mathcal{F}$. Now since for any realization of $\left\{f_{i^{\prime \prime}}\right\}_{i^{\prime \prime}},\left\{x_{i^{\prime}, S}\left(\left\{f_{i^{\prime \prime}}\right\}_{i^{\prime \prime}}\right)\right\}_{i^{\prime}, S}$ satisfy the LP constraints, it follows from linearity of expectation that the expected values $\left\{y_{i^{\prime}, S}\right\}_{i^{\prime}, S}$ also satisfy the constraints. The lemma follows.

For any agent $i^{\prime}$ and item $j$, let $d_{i^{\prime}}^{j}$ be the probability that item $j$ is demanded by agent $i^{\prime}$. That is,

$$
d_{i^{\prime}}^{j}:=\operatorname{Pr}_{f_{i^{\prime}}, g_{-i^{\prime}}}\left[j \in S_{i^{\prime}}\right]=\sum_{S: j \in S} y_{i^{\prime}, S} .
$$

The feasibility of $y_{i^{\prime}, S}$ (Lemma 5) implies: for any $j \in[m]$,

$$
\sum_{i^{\prime} \in[n]} d_{i^{\prime}}^{j}=\sum_{i^{\prime} \in[n], S: j \in S} y_{i^{\prime}, S} \leq 1 .
$$

Also, whether agent $i^{\prime}$ demands $j$ is independent of whether $i^{\prime}$ is served, so for any $j \in[m]$,

$$
\begin{aligned}
\operatorname{Pr}\left[\exists i^{\prime}<i:\left(j \in S_{i^{\prime}} \wedge i^{\prime} \text { is served }\right)\right] & \leq \sum_{i^{\prime}<i} \operatorname{Pr}\left[j \in S_{i^{\prime}} \wedge i^{\prime} \text { is served }\right] \quad \text { (union bound) } \\
& \leq \sum_{i^{\prime} \in[n]} \operatorname{Pr}\left[j \in S_{i^{\prime}} \wedge i^{\prime} \text { is served }\right] \\
& =\sum_{i^{\prime} \in[n]} d_{i^{\prime}}^{j} \cdot \operatorname{Pr}\left[\operatorname{ser}_{i^{\prime}}=1\right] \\
& \leq 1 \cdot q=\frac{1}{2}, \quad \text { (independence of } S_{i^{\prime}} \text { and } \operatorname{ser}_{i^{\prime}} \text { ) }
\end{aligned}
$$

which concludes the proof of the theorem.

\section{The Case of Subadditive Agents}

Equipped with tools developed in previous sections, now we proceed to the case of subadditive agents. Here we choose $q=1$ and

$$
p_{k}= \begin{cases}1 / C, & 1 \leq k \leq C \\ 0, & C<k \leq n,\end{cases}
$$

where $C=\min (100 \log m / \log \log m, n)=O(\log m / \log \log m)$. We prove the following bound for subadditive agents.

Theorem 6. The protocol in Section 3 with the above parameters is $O(\log m / \log \log m)$ competitive when agents are subadditive. 
Proof. When $n \leq 100 \log m / \log \log m$, the theorem is easy to prove. Below we focus on the case where $C=100 \log m / \log \log m<n$. Consider any agent $i$. Fix $f_{i}$ and $S_{i}$. In light of Theorem 2, our goal here is to show

$$
\sum_{1 \leq k \leq C} \mathbb{E}_{R_{i}}\left[f_{i}\left(S_{i}^{k}\right)\right]=\Omega\left(f_{i}\left(S_{i}\right)\right) .
$$

That is, the first $C$ terms in $\left\{f_{i}\left(S_{i}^{k}\right)\right\}_{k \in[n]}$ contribute a constant fraction of $f_{i}\left(S_{i}\right)$ in expectation. The theorem then again follows by plugging the above into Theorem 2, yielding

$$
\mathbb{E}_{R_{i}}\left[u_{i} \mid f_{i}, S_{i}\right] \geq q \cdot \sum_{1 \leq k \leq C} p_{k} \cdot \mathbb{E}_{R_{i}}\left[f_{i}\left(S_{i}^{k}\right)\right]=\Omega\left(f_{i}\left(S_{i}\right) / C\right) .
$$

Taking expectation over $f_{i}$ and $S_{i}$, and summing over the agents, we have

$$
\sum_{i} \mathbb{E}\left[u_{i}\right] \geq \Omega(1 / C) \cdot \sum_{i} \mathbb{E}\left[f_{i}\left(S_{i}\right)\right]=\Omega(1 / C) \cdot \mathrm{LP} \geq \Omega(1 / C) \cdot \mathrm{OPT} .
$$

A competitive ratio of $O(C)=O(\log m / \log \log m)$ follows. The rest of the proof is dedicated to establishing (2) when $i, f_{i}$ and $S_{i}$ are fixed.

The plan is to show, with constant probability over $R_{i}, S_{i}^{k}=\emptyset$ for all $k>C$. Denote this event by $\mathcal{E}_{i}$. Whenever this happens, $\bigcup_{1 \leq k \leq C} S_{i}^{k}=S_{i}$, and by the subadditivity of $f_{i}$,

$$
\sum_{1 \leq k \leq C} f_{i}\left(S_{i}^{k}\right) \geq f_{i}\left(S_{i}\right)
$$

As a result,

$$
\sum_{1 \leq k \leq C} \mathbb{E}_{R_{i}}\left[f_{i}\left(S_{i}^{k}\right)\right] \geq \sum_{1 \leq k \leq C} \mathbb{E}_{R_{i}}\left[f_{i}\left(S_{i}^{k}\right) \mid \mathcal{E}_{i}\right] \cdot \operatorname{Pr}\left[\mathcal{E}_{i}\right] \geq \operatorname{Pr}\left[\mathcal{E}_{i}\right] \cdot f_{i}\left(S_{i}\right) .
$$

We show below $\operatorname{Pr}\left[\mathcal{E}_{i}\right]=\Omega(1)$.

Fix an item $j$, and consider the probability that $j \in S_{i}^{k}$ for some $k>C$, or equivalently,

$$
\operatorname{Pr}\left[\sum_{i^{\prime}<i} \mathbb{I}\left[j \in S_{i^{\prime}}\right] \geq C\right] .
$$

To bound the above probability, recall the following fact about independent Bernoulli variables (see, e.g., $[14,19])$.

- Lemma 7. For any $n \in \mathbb{Z}^{+}$, independent Bernoulli random variables $X_{1}, \ldots, X_{n}$ where $\mathbb{E}\left[\sum_{i \in[n]} X_{i}\right] \leq 1$, and any $k \in \mathbb{Z}^{+}$,

$$
\operatorname{Pr}\left[\sum_{i} X_{i} \geq k\right]=O\left(\frac{1}{k !}\right)=k^{-\Omega(k)} .
$$

The lemma says, that if the sum of independent Bernoulli variables in expectation does not exceed 1 , then the tail of this sum decays factorially fast. Now observe that $S_{1}, \ldots, S_{i-1}$, and therefore $\mathbb{I}\left[j \in S_{1}\right], \ldots, \mathbb{I}\left[j \in S_{i-1}\right]$ are independent. This is because for any $i^{\prime}, S_{i^{\prime}}$ depends only on $f_{i^{\prime}}$ and $g_{-i^{\prime}}$. Also, since

$$
\mathbb{E}\left[\sum_{i^{\prime}<i} \mathbb{I}\left[j \in S_{i^{\prime}}\right]\right]=\sum_{i^{\prime}<i} \operatorname{Pr}\left[j \in S_{i^{\prime}}\right]=\sum_{i^{\prime}<i} d_{j}^{i^{\prime}} \leq \sum_{i^{\prime} \in[n]} d_{j}^{i^{\prime}} \leq 1,
$$


random variables $\left\{\mathbb{I}\left[j \in S_{i^{\prime}}\right]\right\}_{i^{\prime}<i}$ satisfy the conditions of Lemma 7 . So for large enough $m$, we can bound the probability that $j \in S_{i}^{k}$ for some $k>C$ using Lemma 7 . Recall that $C=100 \log m / \log \log m$. Plugging this in, Lemma 7 then gives

$$
\operatorname{Pr}\left[\sum_{i^{\prime}<i} \mathbb{I}\left[j \in S_{i^{\prime}}\right] \geq C\right] \leq C^{-\Omega(C)}=O\left(\frac{1}{m^{2}}\right) .
$$

Now taking a union bound over all items, we get

$$
\operatorname{Pr}\left[\mathcal{E}_{i}\right] \geq 1-\sum_{j \in[m]} \operatorname{Pr}\left[\sum_{i^{\prime}<i} \mathbb{I}\left[j \in S_{i^{\prime}}\right] \geq C\right]=1-\sum_{j \in[m]} O\left(\frac{1}{m^{2}}\right)=1-O\left(\frac{1}{m}\right)=\Omega(1) .
$$

This concludes the proof of the theorem.

\section{Generalizing to Approximately Subadditive Agents}

In this section, we consider valuations with superadditive width at most $D>0$. In order to utilize the boundedness of the superadditive width, we set $q=\frac{1}{4 D}$, and

$$
p_{k}= \begin{cases}\frac{1}{2}+\frac{1}{2 C}, & k=1 \\ \frac{1}{2 C}, & 1<k \leq C \\ 0, C<k \leq n, & \end{cases}
$$

where again $C=\min (100 \log m / \log \log m, n)=O(\log m / \log \log m)$. One may check that $\left\{p_{k}\right\}_{k \in[n]}$ in fact sum to 1 . We prove the following competitive ratio.

- Theorem 8. The protocol in Section 3 with the above parameters is $O(D \log m / \log \log m)$ competitive when all valuations have superadditive width at most $D$.

Proof. Again, fix an agent $i, f_{i}$ and $S_{i}$. Our goal is to show that one of the following two claims is always true:

- Claim (i):

$$
\mathbb{E}_{R_{i}}\left[f_{i}\left(S_{i}^{1}\right)\right]=\Omega\left(f_{i}\left(S_{i}\right) / C\right) .
$$

- Claim (ii):

$$
\sum_{1 \leq k \leq C} \mathbb{E}_{R_{i}}\left[f_{i}\left(S_{i}^{k}\right)\right]=\Omega\left(f_{i}\left(S_{i}\right)\right)
$$

We first show how the above condition implies the theorem. Again, by applying Theorem 2,

$$
\begin{aligned}
\mathbb{E}_{R_{i}}\left[u_{i} \mid f_{i}, S_{i}\right] & \geq q \cdot \sum_{1 \leq k \leq C} p_{k} \cdot \mathbb{E}_{R_{i}}\left[f_{i}\left(S_{i}^{k}\right)\right] \\
& =\Omega(1 / D) \cdot\left(\mathbb{E}_{R_{i}}\left[f_{i}\left(S_{i}^{k}\right)\right]+\frac{1}{C} \sum_{1 \leq k \leq C} \mathbb{E}_{R_{i}}\left[f_{i}\left(S_{i}^{k}\right)\right]\right) \\
& =\Omega(1 / D) \cdot \Omega\left(f_{i}\left(S_{i}\right) / C\right) \\
& =\Omega\left(\frac{D \log m}{\log \log m} \cdot f_{i}\left(S_{i}\right)\right) .
\end{aligned}
$$

The theorem then follows by taking expectation over $f_{i}$ and $S_{i}$, and summing over the agents. 
The rest of the proof is dedicated to establishing the above condition. We consider the most valuable $2 D$ items for agent $i$ in $S_{i}$, i.e.,

$$
S_{i}^{*}:=\operatorname{argmax}_{S: S \subseteq S_{i},|S| \leq 2 D} f_{i}(S) .
$$

We show below that when the value of $S_{i}^{*}, f_{i}\left(S_{i}^{*}\right)$, is small, then Claim (i) holds. Otherwise, Claim (ii) holds. Since $f_{i}$ and $S_{i}$ are fixed, one of the two claims is always true.

First suppose

$$
f_{i}\left(S_{i}^{*}\right) \geq f_{i}\left(S_{i}\right) /(2 C) .
$$

In such cases, we show that with constant probability, $S_{i}^{*} \subseteq S_{i}^{1}$. Whenever this happens, monotonicity implies

$$
f_{i}\left(S_{i}^{1}\right) \geq f_{i}\left(S_{i}^{*}\right) \geq f_{i}\left(S_{i}\right) /(2 C) .
$$

Taking expectation over $R_{i}$, this implies Claim (i).

We now bound the probability that $S_{i}^{*} \subseteq S_{i}^{1}$. Fix $j \in S_{i}^{*}$. Consider the probability that $j \notin S_{i}^{1}$, or equivalently,

$$
\operatorname{Pr}\left[\sum_{i^{\prime}<i} \mathbb{I}\left[j \in S_{i^{\prime}} \wedge i^{\prime} \text { is served }\right] \geq 1\right] .
$$

We upper bound this probability in the following way.

$$
\begin{aligned}
\operatorname{Pr}\left[\sum_{i^{\prime}<i} \mathbb{I}\left[j \in S_{i^{\prime}} \wedge i^{\prime} \text { is served }\right] \geq 1\right] & \leq \sum_{i^{\prime}<i} \operatorname{Pr}\left[j \in S_{i^{\prime}} \wedge i^{\prime} \text { is served }\right] \quad \text { (union bound) } \\
& =\sum_{i^{\prime}<i} d_{i^{\prime}}^{j} \cdot q \leq \sum_{i^{\prime} \in[n]} d_{i^{\prime}}^{j} \cdot q \\
& \leq q=\frac{1}{4 D} .
\end{aligned}
$$

So for any $j \in S_{i}^{*}$,

$$
\operatorname{Pr}\left[j \notin S_{i}^{1}\right] \leq \frac{1}{4 D} .
$$

Since $\left|S_{i}^{*}\right|=2 d$, taking a union bound over all items in $S_{i}^{*}$, we get

$$
\operatorname{Pr}\left[S_{i}^{*} \subseteq S_{i}^{1}\right] \geq 1-\sum_{j \in S_{i}^{*}} \operatorname{Pr}\left[j \notin S_{i}^{1}\right] \geq 1-2 D \cdot \frac{1}{4 D}=\frac{1}{2} .
$$

This implies Claim (i) as argued above.

Now suppose

$$
f_{i}\left(S_{i}^{*}\right)<f_{i}\left(S_{i}\right) /(2 C) \text {. }
$$

Given this, we show Claim (ii) holds. First we need the following property of valuations with bounded superadditive width.

- Lemma 9 ([7]). Let $f: 2^{[m]} \rightarrow \mathbb{R}_{+}$be a valuation function such that $\mathrm{SAW}(f) \leq D$. For any $S, T \subseteq[m]$,

$$
f(S \mid T) \leq \min _{T^{\prime}: T^{\prime} \subseteq T,\left|T^{\prime}\right| \leq D} f\left(S \mid T^{\prime}\right) .
$$


Consider the prefix unions of $\left\{S_{i}^{k}\right\}_{k \in[C]}$. To be precise, let $U_{i}^{0}=\emptyset$, and for any $k \in[C]$,

$$
U_{i}^{k}=U_{i}^{k-1} \cup S_{i}^{k} .
$$

Recall that for any $S, T \subseteq[m], f_{i}(S \mid T)=f_{i}(S \cup T)-f_{i}(T)$ is the marginal value of $S$ given $T$. For any $k \in[C]$, we have

$$
\begin{aligned}
& f_{i}\left(U_{i}^{k}\right)=f_{i}\left(S_{i}^{k} \mid U_{i}^{k-1}\right)+f_{i}\left(U_{i}^{k-1}\right) \\
& \leq f_{i}\left(S_{i}^{k} \mid X\right)+f_{i}\left(U_{i}^{k-1}\right) \text {, where } X=\operatorname{argmin}_{S: S \subseteq U_{i}^{k-1},|S| \leq D} f_{i}\left(S_{i}^{k} \mid S\right) \\
& \leq f_{i}\left(S_{i}^{k} \cup X\right)+f_{i}\left(U_{i}^{k-1}\right) \\
& =f_{i}\left(X \mid S_{i}^{k}\right)+f_{i}\left(S_{i}^{k}\right)+f_{i}\left(U_{i}^{k-1}\right) \\
& \leq f_{i}(X \mid Y)+f_{i}\left(S_{i}^{k}\right)+f_{i}\left(U_{i}^{k-1}\right) \text {, where } Y=\operatorname{argmin}_{S: S \subseteq S_{i}^{k},|S| \leq D} f_{i}(X \mid S) \\
& \leq f_{i}(X \cup Y)+f_{i}\left(S_{i}^{k}\right)+f_{i}\left(U_{i}^{k-1}\right) \\
& \leq f_{i}\left(S_{i}^{*}\right)+f_{i}\left(S_{i}^{k}\right)+f_{i}\left(U_{i}^{k-1}\right) \text {. (definition of } S_{i}^{*} \text { and }|X \cup Y| \leq|X|+|Y| \leq 2 D \text { ) }
\end{aligned}
$$

Now recall that $\mathcal{E}_{i}$ is the event that for all $k>C, S_{i}^{k}=\emptyset$. In the proof of Theorem 6, we have shown that $\operatorname{Pr}\left[\mathcal{E}_{i}\right]=\Omega(1)$. Also, whenever $\mathcal{E}_{i}$ happens, we have $U_{i}^{C}=S_{i}$. As a result, when $\mathcal{E}_{i}$ happens, we can bound $f_{i}\left(S_{i}\right)$ in the following way.

$$
\begin{aligned}
f_{i}\left(S_{i}\right)=f_{i}\left(U_{i}^{C}\right) & \leq f_{i}\left(S_{i}^{*}\right)+f_{i}\left(S_{i}^{C}\right)+f_{i}\left(U_{i}^{C-1}\right) \\
& \leq 2 f_{i}\left(S_{i}^{*}\right)+f_{i}\left(S_{i}^{C}\right)+f_{i}\left(S_{i}^{C-1}\right)+f_{i}\left(U_{i}^{C-2}\right) \\
& \leq \ldots \\
& \leq C \cdot f_{i}\left(S_{i}^{*}\right)+\sum_{1 \leq k \leq C} f_{i}\left(S_{i}^{k}\right) .
\end{aligned}
$$

Compared to the subadditive case, here we have the additional term $C \cdot f_{i}\left(S_{i}^{*}\right)$. However, as we are in the world where $f_{i}\left(S_{i}^{*}\right)$ is small, this term does not affect the bound too much. Concretely, whenever $\mathcal{E}_{i}$ happens, we have

$$
\sum_{1 \leq k \leq C} f_{i}\left(S_{i}^{k}\right) \geq f_{i}\left(S_{i}\right)-C \cdot f_{i}\left(S_{i}^{*}\right)>f_{i}\left(S_{i}\right)-C \cdot \frac{f_{i}\left(S_{i}\right)}{2 C}=\frac{f_{i}\left(S_{i}\right)}{2} .
$$

Again, since $\operatorname{Pr}\left[\mathcal{E}_{i}\right]=\Omega(1)$, we have

$$
\sum_{1 \leq k \leq C} \mathbb{E}_{R_{i}}\left[f_{i}\left(S_{i}^{k}\right)\right]=\Omega\left(f_{i}\left(S_{i}\right)\right)
$$

which is precisely Claim (ii), and therefore concludes the proof of the theorem.

\section{- References}

1 Saeed Alaei. Bayesian combinatorial auctions: Expanding single buyer mechanisms to many buyers. SIAM Journal on Computing, 43(2):930-972, 2014.

2 Kshipra Bhawalkar and Tim Roughgarden. Welfare guarantees for combinatorial auctions with item bidding. In Proceedings of the twenty-second annual ACM-SIAM symposium on Discrete Algorithms, pages 700-709. Society for Industrial and Applied Mathematics, 2011.

3 Liad Blumrosen and Thomas Holenstein. Posted prices vs. negotiations: an asymptotic analysis. In $E C$, page 49. Citeseer, 2008. 
4 Yang Cai and Mingfei Zhao. Simple mechanisms for subadditive buyers via duality. In Proceedings of the 49th Annual ACM SIGACT Symposium on Theory of Computing, pages 170-183. ACM, 2017.

5 Shuchi Chawla, Jason D Hartline, and Robert Kleinberg. Algorithmic pricing via virtual valuations. In Proceedings of the 8th ACM conference on Electronic commerce, pages 243-251. ACM, 2007.

6 Shuchi Chawla, Jason D Hartline, David L Malec, and Balasubramanian Sivan. Multiparameter mechanism design and sequential posted pricing. In Proceedings of the forty-second ACM symposium on Theory of computing, pages 311-320. ACM, 2010.

7 Wei Chen, Shang-Hua Teng, and Hanrui Zhang. Capturing complementarity in set functions by going beyond submodularity/subadditivity. 10th Innovations in Theoretical Computer Science, 2019.

8 Vincent Cohen-Addad, Alon Eden, Michal Feldman, and Amos Fiat. The invisible hand of dynamic market pricing. In Proceedings of the 2016 ACM Conference on Economics and Computation, pages 383-400. ACM, 2016.

9 Shahar Dobzinski, Noam Nisan, and Michael Schapira. Approximation algorithms for combinatorial auctions with complement-free bidders. In Proceedings of the thirty-seventh annual ACM symposium on Theory of computing, pages 610-618. ACM, 2005.

10 Paul Dütting, Michal Feldman, Thomas Kesselheim, and Brendan Lucier. Prophet inequalities made easy: Stochastic optimization by pricing non-stochastic inputs. In Foundations of Computer Science (FOCS), 2017 IEEE 58th Annual Symposium on, pages 540-551. IEEE, 2017.

11 Paul Dütting, Thomas Kesselheim, and Brendan Lucier. An o (log log $\mathrm{m}$ ) prophet inequality for subadditive combinatorial auctions. arXiv preprint, 2020. arXiv:2004.09784.

12 Paul Dütting and Robert Kleinberg. Polymatroid prophet inequalities. In Algorithms-ESA 2015, pages 437-449. Springer, 2015.

13 Soheil Ehsani, MohammadTaghi Hajiaghayi, Thomas Kesselheim, and Sahil Singla. Prophet secretary for combinatorial auctions and matroids. In Proceedings of the Twenty-Ninth Annual ACM-SIAM Symposium on Discrete Algorithms, pages 700-714. SIAM, 2018.

14 Uriel Feige. On maximizing welfare when utility functions are subadditive. SIAM Journal on Computing, 39(1):122-142, 2009.

15 Uriel Feige, Michal Feldman, Nicole Immorlica, Rani Izsak, Brendan Lucier, and Vasilis Syrgkanis. A unifying hierarchy of valuations with complements and substitutes. In TwentyNinth AAAI Conference on Artificial Intelligence, 2015.

16 Michal Feldman, Nick Gravin, and Brendan Lucier. Combinatorial auctions via posted prices. In Proceedings of the twenty-sixth annual ACM-SIAM symposium on Discrete algorithms, pages 123-135. Society for Industrial and Applied Mathematics, 2015.

17 Moran Feldman, Ola Svensson, and Rico Zenklusen. A simple $O(\log \log (\operatorname{rank}))$-competitive algorithm for the matroid secretary problem. In Proceedings of the twenty-sixth annual ACM-SIAM symposium on Discrete algorithms, pages 1189-1201. SIAM, 2014.

18 Mohammad Taghi Hajiaghayi, Robert Kleinberg, and Tuomas Sandholm. Automated online mechanism design and prophet inequalities. In $A A A I$, volume 7, pages 58-65, 2007.

19 Svante Janson. Large deviation inequalities for sums of indicator variables. arXiv preprint, 2016. arXiv: 1609.00533.

20 Robert Kleinberg and Seth Matthew Weinberg. Matroid prophet inequalities. In Proceedings of the forty-fourth annual ACM symposium on Theory of computing, pages 123-136. ACM, 2012.

21 Ulrich Krengel and Louis Sucheston. Semiamarts and finite values. Bulletin of the American Mathematical Society, 83(4):745-747, 1977.

22 Ulrich Krengel and Louis Sucheston. On semiamarts, amarts, and processes with finite value. Probability on Banach spaces, 4:197-266, 1978. 
23 Brendan Lucier. An economic view of prophet inequalities. ACM SIGecom Exchanges, 16(1):24-47, 2017.

24 Noam Nisan and Ilya Segal. The communication requirements of efficient allocations and supporting prices. Journal of Economic Theory, 129(1):192-224, 2006.

25 Aviad Rubinstein and Sahil Singla. Combinatorial prophet inequalities. In Proceedings of the Twenty-Eighth Annual ACM-SIAM Symposium on Discrete Algorithms, pages 1671-1687. SIAM, 2017. 